\title{
Fisher information of special functions and second-order differential equations
}

\author{
R.J. Yáñez ${ }^{a, b}$, P. Sánchez-Moreno ${ }^{a, c, *}$, J.S. Dehesa ${ }^{a, c}$ \\ ${ }^{a}$ Instituto Carlos I de Física Teórica y Computacional, University of Granada, \\ Granada, Spain \\ ${ }^{\mathrm{b}}$ Departamento de Matemática Aplicada, University of Granada, Granada, Spain \\ ${ }^{\mathrm{c}}$ Departamento de Física Atómica, Molecular y Nuclear, University of Granada, \\ Granada, Spain
}

\begin{abstract}
We investigate a basic question of information theory, namely the evaluation of the Fisher information and the relative Fisher information with respect to a nonnegative function, for the probability distributions obtained by squaring the special functions of mathematical physics which are solutions of second-order differential equations. Emphasis is made in the Nikiforov-Uvarov hypergeometric-type functions. We obtain explicit expressions for these information-theoretic properties via the expectation values of the coefficients of the differential equation. We illustrate our approach for various special functions of physico-mathematical interest.
\end{abstract}

Key words: Special functions, Information theory, Fisher information, Differential equations, Hypergeometric-type equations, Classical orthogonal polynomials 2000 MSC: 42C05, 33C45, 94A17, 34G10, 81Q05

\section{Introduction}

Special functions of applied mathematics and mathematical physics usually arise as solutions of differential equations, which are in practice the natural mathematical formulations of an enormous amount of physical problems [36].

* Corresponding author. Address: Departamento de Física Atómica, Molecular y Nuclear, University of Granada, Avda. Fuentenueva S/N, E-18071, Granada, Spain Email addresses: ryanez@ugr.es (R.J. Yáñez), pablos@ugr.es (P. Sánchez-Moreno), dehesa@ugr.es (J.S. Dehesa). 
Often these equations have the following second order form

$$
u^{\prime \prime}+a(x) u^{\prime}+b(x) u=0
$$

where $a(x)$ and $b(x)$ are functions with appropriate regularity conditions. Most of the exactly solvable fundamental wave equations are (or can be transformed into) particular cases of Eq. (1) with the form

$$
u^{\prime \prime}+\frac{\tilde{\tau}(x)}{\sigma(x)} u^{\prime}+\frac{\tilde{\sigma}(x)}{\sigma^{2}(x)} u=0
$$

where $\sigma(x)$ and $\tilde{\sigma}(x)$ are polynomials of, at most, second degree, and $\tilde{\tau}(x)$ is a polynomial of, at most, first degree. This is the generalized equation of hypergeometric type [36], also called Fuchs or, most appropriately, Nikiforov-Uvarov equation. Non-relativistic and relativistic quantum-mechanical equations of motion (e.g. Schrödinger, Klein-Gordon, Dirac) for single-particle systems in spherically symmetric potentials with arbitrary dimensions, as well as equations which describe numerous physical phenomena of many-fermion systems (atoms, molecules, nuclei) and some classical wave equations (e.g. Laplace, Helmholtz) have this form $[2,22,36]$.

The special functions which occur in classical and quantum physics (e.g., hypergeometric functions, confluent hypergeometric functions, spherical harmonics, classical orthogonal polynomials) often belong to the large family of generalized hypergeometric-type functions, to be referred heretoforth as NikiforovUvarov functions to honour the powerful and unifying theoretical ideas and techniques to handle these objects; that is, they are solutions of second order differential equations of the form (2). Let us realize that when $\sigma(x)$ is a quadratic polynomial, Eq. (2) is a special case of the Riemann equation with three different singular points, one of them at infinity [34,56]. Moreover by appropriate changes of the dependent variable $u(x) \rightarrow y(x)$, Eq. (2) can be transformed into the simpler hypergeometric-type equation [36]

$$
\sigma(x) y^{\prime \prime}+\tau(x) y^{\prime}+\lambda y=0
$$

where $\tau(x)$ is a polynomial of, at most, first degree and $\lambda$ is a constant. The socalled hypergeometric functions and the confluent hypergeometric functions are particular hypergeometric-type functions with $\sigma(x)=x(1-x)$ and $\sigma(x)=$ $x$, respectively.

Then, a natural approach for mathematical physics is to obtain the algebraic properties of the special functions (and so, in particular, the stationary quantum-mechanical wavefunctions of physical systems) directly from the differential equations (1), (2) and (3) that they satisfy. This approach has been pioneered by Arnold F. Nikiforov and Vasilii B. Uvarov [36] who developed an elegant technique to determine numerous algebraic properties of 
the hypergeometric-type functions and some generalized hypergeometric-type functions. This technique, which works when the polynomial coefficients $\sigma(x)$ and $\tau(x)$ do not depend on the spectral parameter $\lambda$ [58], was later on developed and extended to find linear and non-linear recurrence relations for hypergeometric-type functions with three and four terms $[17-19,58,59]$ and applied to some quantum-mechanical problems [3,4]. Just recently, it has been generalized to the case when the polynomial coefficients of Eq. (3) do depend on $\lambda$ [60]; this case corresponds, in fact, to the real physical wave equations. The technique of Zarzo et al. [60] has not yet been, however, sufficiently well explored from both fundamental and applied standpoints. For completeness, let us also mention the recent criterion of Saad et al [46] for the polynomial solutions of Eq. (1) and the very interesting and useful theory of the orthogonal polynomial solutions of higher order differential and partial differential equations of Littlejohn et al. [20,31], as well as their classification based on Lie-algebras ideas $[27,53]$.

In this paper we continue to develop the following programme: To calculate the information-theoretic properties of the solutions of Eqs. (1), (2) and (3) from their corresponding coefficients. This programme includes the determination of the information-theoretic measures, such as the Fisher information and the Shannon, Renyi and Tsallis entropies, which quantify the spreading of these functions in a complementary and more appropriate manner than the familiar variance. These quantities are defined by the corresponding spreading measures of the probability distribution obtained by squaring the associated special functions. This programme was initiated in Ref. [48] with the calculation of the Fisher information of the classical orthogonal polynomials, which are solutions of Eq. (3), and then the corresponding Cramer-Rao information plane [16] was analyzed. See also [15], where the parameter-based Fisher information of classical orthogonal polynomials was calculated by use of their algebraic properties other than the differential equation. Now, our purpose is the analytic evaluation of the Fisher information of special functions other than the classical orthogonal polynomials, in terms of the expectation values of the coefficients which characterize the differential equations (1), (2) or (3) that they satisfy: This is the main goal of this work. Furthermore, we apply the resulting general expressions to various specific second order differential equations with standard and non-standard dimensions. In doing this for the Schrödinger equation of particles moving in a $D$-dimensional central potential we realize the convenience of introducing a generalized Fisher information , which then is calculated in terms of the given quantum-mechanical potential.

The Fisher information, firstly used for statisticians and electrical engineers and later on by quantum physicists [24], does not only measure the spreading of the solutions of the differential equations in a complementary and qualitatively different manner as the familiar variance and the global information entropies (Shannon, Renyi, Tsallis), but also it quantifies their oscillatory character. 
The structure of the paper is the following. First in Section 2 the notions of Fisher and relative Fisher information of a probability distribution are discussed and some properties and applications are shown to gain a deeper insight into them. Then, in Section 3 we find the explicit expressions of the Fisher informations of the differential equations (1), (2) and (3) in terms of the expectation values of their coefficients, and we apply them to some particular equations of relevant physico-mathematical interest. In Section 4 the relative Fisher information of some Nikiforov-Uvarov functions which arise in the study of some $D$-dimensional quantum-mechanical systems and play an important role in applied mathematics (hypergeometric-type functions) are explicitly found. Finally, some conclusions and open problems are given.

\section{Fisher's information of a probability density}

Here we define the Fisher information of a probability density and the relative Fisher information between two probability densities, and we point out some of their properties relevant for the purposes of this work and its possible extension.

\subsection{The Fisher information}

The concept of Fisher information of a probability density and its basic properties, which are not yet sufficiently well known in spite of their early origin [21] and the increasing number of its applications in the last few years $[7-9,14,16,24,26,28,29,33,35,40,42,44,45,48,52]$. The translationally invariant Fisher information of a probability density $\rho(x)$ with support interval $\Delta \in \mathbb{R}$ is defined as the expectation value of the squared logarithmic derivative of $\rho(x)$; that is,

$$
I(\rho):=\left\langle\left[\frac{d}{d x} \log \rho(x)\right]^{2}\right\rangle=\int_{\Delta} \frac{\left[\rho^{\prime}(x)\right]^{2}}{\rho(x)} d x,
$$

where we have used the following notation

$$
\langle f(x)\rangle:=\int_{\Delta} \rho(x) f(x) d x
$$

for the expectation value of the function $f(x)$. Up until 2003, the monograph of Frieden [24] gives an almost exhaustive account of this information-theoretical notion, its properties and its applications to an enormous amount of natural phenomena and scientific fields. 
For brevity, let us only mention that, according to Eq. (4), the Fisher information is a measure of the derivative (gradient) content of the probability density; so, when $\rho(x)$ has a discontinuity, the local slope value changes drastically and then the Fisher information strongly alters. This indicates that it is a local quantity in contrast to the variance $V(\rho)$ and other informationtheoretic quantities, such as the Shannon entropy $H(\rho):=-\int \rho(x) \ln \rho(x) d x$, which have a global character because they are powerlike and logarithmic functionals of the density, respectively. These three notions are mutually related via the elegant de Bruijn identity [6] and the so-called Cramer-Rao [52] and Shannon inequalities given by

$$
\begin{aligned}
& V(\rho) I(\rho) \geq 1, \\
& N(\rho) I(\rho) \geq 1, \text { with } N(\rho):=\frac{1}{2 \pi e} \exp [2 H(\rho)]
\end{aligned}
$$

respectively. Further inequalities relating the Fisher information with other power and logarithmic expectation values of $\rho(x)$ have been variationally found [41].

Moreover, the Fisher information has been shown to be a measure of disorder or smoothness of the probability density $\rho(x)$ and uncertainty of the associated random variable $X$. The disorder aspect has been discussed in depth by Frieden $[23,24]$, and the uncertainty properties are clearly shown by the Stam inequalities [52] and the recently discovered Fisher-information-based uncertainty relation $[7,14]$

$$
I(\rho) I(\tilde{\rho}) \geq 4
$$

where $\tilde{\rho}$ denotes the probability density associated to the Fourier transform of the variable $X$. For a deeper understanding of the Fisher notion, let us point out that broad and smooth densities have low gradient contents and so their Fisher information is small. Conversely, if $\rho(x)$ shows an undue preference or bias towards particular $x$ values, then it is steeply sloped about these $x$ values, and so the value of the Fisher information becomes large. Then, a largely uniform or unbiased density exhibits large uncertainty (high disorder), which means lack of predictability of values $x$ over its range of validity, and a peaked or biased density to particular $x$ values exhibits small uncertainty (low disorder).

The Fisher information has been evaluated in a closed analytical form for various probability distributions $[6,55]$ : the Gaussian, the Student-t and some power exponential distributions. It is worth noticing that $I\left(\rho_{U}\right)=0$ for the uniform distribution 


$$
\rho_{U}(x)= \begin{cases}1 / a, & 0<x<a \\ 0, & \text { elsewhere }\end{cases}
$$

and $I\left(\rho_{G}\right)=1 / \sigma^{2}$ for the Gaussian distribution with finite variance $V\left(\rho_{G}\right)=$ $\sigma^{2}$ given by

$$
\rho_{G}(x)=\frac{1}{\sqrt{2 \pi \sigma^{2}}} \exp \left[-\frac{(x-\mu)^{2}}{\sigma^{2}}\right] ; x \in(-\infty,+\infty) .
$$

The last observation, which is true for a general law $\rho(x)$ (provided, of course, that its variance be finite) due to the proportionality to the square derivative of $\rho(x)$ in Eq. (4), indicates that the broader and smoother $\rho(x)$, the smaller $I(\rho)$ is. Moreover, it is most interesting for the purposes of this work to realize that the Chebyshev sinusoidal distributions

$$
\rho_{S}(x)=\frac{1}{\pi} \sin ^{2} n x ; x \in[-\pi, \pi], n=1,2, \ldots
$$

and

$$
\tilde{\rho}_{S}(x)=\frac{1}{2 n \pi} \frac{\sin ^{2}(n x)}{\sin ^{2} x} ; x \in[-\pi, \pi], n=1,2, \ldots,
$$

have the Fisher information

$$
I\left(\rho_{S}\right)=4 n^{2}
$$

and

$$
I\left(\tilde{\rho}_{S}\right)=\frac{4}{3}\left(n^{2}-1\right),
$$

respectively, indicating that the more oscillatory $\rho(x)$, the bigger $I(\rho)$ is.

For further details about the Fisher information, its properties and applications see Refs. [6-10, 14, 15, 23, 24, 26, 28-30, 33, 35, 40-42, 44, 45, 50, 52, 55]. Let us finally remark that this quantity has been used (i) to describe, a factor apart, various macroscopic quantities, e.g. the kinetic [33, 41, 43,50] and Weissäcker $[40,41,43]$, (ii) to derive the Schrödinger and Klein-Gordon equations of motion $[24,39]$ as well as the Euler equation of the density functional theory [35] from the principle of minimum Fisher information, (iii) to predict the most distinctive non-linear phenomena (avoided crossings) encountered in atoms and molecules under strong external fields [26], and (iv) to measure the non-classicality of quantum systems $[28,29]$, and (v) to obtain the foundations of thermodynamics in a microscopic way $[25,37]$. 


\subsection{The relative Fisher information}

The Fisher information of the probability density $\rho_{1}(x)$ relative with respect to the probability density $\rho_{2}(x)$ is defined by

$$
I\left[\rho_{1}, \rho_{2}\right]=\int \rho_{1}(x)\left[\frac{d}{d x} \log \frac{\rho_{1}(x)}{\rho_{2}(x)}\right]^{2} d x .
$$

This non-negative quantity expresses somehow a difference of information. It expresses when the two densities approach each other, and vanishes when they are equal.

The particular case $\rho_{1}(x) / \rho_{2}(x)=\rho(x)$, where $\rho(x)$ is itself a probability density (so that $\rho_{1}(x)=\rho(x) \omega(x)$ and $\rho_{2}(x)=\omega(x)$ ) is of special importance for applied mathematicians and mathematical physicists, as it is illustrated later in Section 4. Then, the relative Fisher information between $\rho_{1}(x)$ and $\omega(x)$ is given by

$$
\begin{aligned}
I_{\omega}[\rho] & \equiv I[\rho \omega, \omega]=\int \rho(x)\left[\frac{d}{d x} \log \rho(x)\right]^{2} \omega(x) d x \\
& =\int \frac{\left[\rho^{\prime}(x)\right]^{2}}{\rho(x)} \omega(x) d x .
\end{aligned}
$$

Let us remark that for $\omega(x)=1$ one has, because of (4), that $I_{1}[\rho]=I[\rho]$; so, the standard Fisher information gives somehow the separation of the probability density out from uniformity, indicating that it is a measure of disorder as already pointed out.

To gain more insight into the notion of relative Fisher information of two distributions, let us calculate it for the two following particular cases:

(a) Fisher information between two Freud-like probability densities given by

$$
\rho_{\mu}(x)=\frac{1}{\Gamma\left(1+\frac{1}{\mu}\right)} e^{-x^{\mu}} ; x \in[0, \infty), \mu>0 .
$$

These distributions with arbitrary non-negative parameter play an important role in numerous scientific fields, from communication engineering [57] to the theory of special functions in applied mathematics and mathematical physics [54]. From Eq. (8) we obtain that

$$
I\left(\rho_{\mu}, \rho_{\nu}\right)=\frac{1}{\Gamma(1 / \mu)}\left[\mu^{2} \Gamma\left(2-\frac{1}{\mu}\right)-2 \mu \nu \Gamma\left(\frac{\mu+\nu+1}{\mu}\right)+\nu^{2} \Gamma\left(\frac{2 \nu-1}{\mu}\right)\right] ; \mu>\frac{1}{2},
$$




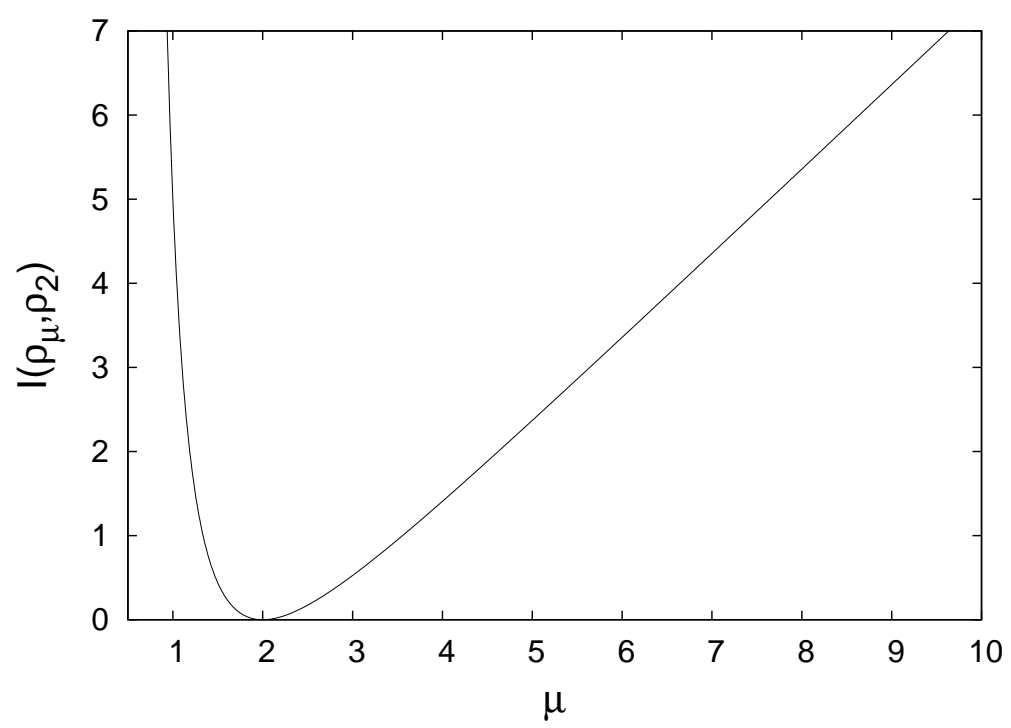

Fig. 1. $I\left(\rho_{\mu}, \rho_{2}\right)$ for the Freud-like class as a function of $\mu$.

for the Fisher information between two arbitrary members $(\mu, \nu)$ of the Freud-like class (9), and

$$
I\left(\rho_{\mu}, \rho_{2}\right)=\frac{1}{\Gamma(1 / \mu)}\left[\mu^{2} \Gamma\left(2-\frac{1}{\mu}\right)-4 \mu \Gamma\left(1+\frac{1}{\mu}\right)+4 \Gamma\left(\frac{3}{\mu}\right)\right],
$$

for the Fisher information of any Freud-like density $\rho_{\mu}(x)$ with respect to the Gaussian density $\rho_{2}(x)$. It is worth noticing that the relative Fisher information $I\left(\rho_{\mu}, \rho_{\nu}\right)$ vanishes when the non-negative parameters are equal.

In Figure 1 we have plotted the relative Fisher $I\left(\rho_{\mu}, \rho_{2}\right)$ with respect to $\mu$. We find that this quantity has an asymmetric parabolic behaviour with a minimum equal to zero at $\mu=2$, as expected since then the two involved densities are equal. For $\frac{1}{2}<\mu \leq 2$ the Freud-like density approaches very fast to the Gaussian density, while for $\mu>2$ the Freud-like density goes monotonically and almost linerly away from the Gaussian.

(b) Fisher information between the Jacobi-like probability density given by

$$
\rho_{\alpha, \beta}(x)=\frac{(1-x)^{\alpha}(1+x)^{\beta}}{2^{\alpha+\beta+1} B(\alpha+1, \beta+1)} ; x \in[-1,1], \alpha, \beta>-1,
$$

and the Chebyshev density of first type $\rho_{C}(x) \equiv \rho_{-1 / 2,-1 / 2}(x)=\pi^{-1}(1-$ $\left.x^{2}\right)^{-1 / 2}$. We have obtained for this quantity the value

$$
\begin{aligned}
& I\left(\rho_{\alpha, \beta}, \rho_{C}\right) \\
= & \frac{(\alpha+\beta)(\alpha+\beta+1)\left(\alpha^{2}(4 \beta+5)+\alpha(2 \beta(2 \beta-5)-3)+(\beta-1)(5 \beta+2)\right)}{16 \alpha \beta(\alpha-1)(\beta-1)} ; \alpha, \beta>1,
\end{aligned}
$$

which is symmetric under the interchange between $\alpha$ and $\beta$. In Figure 2 this quantity is represented as a function of $\alpha$ for $\beta=2$. Here again we observe an asymmetric parabolic behaviour with a non-vanishing minimum 


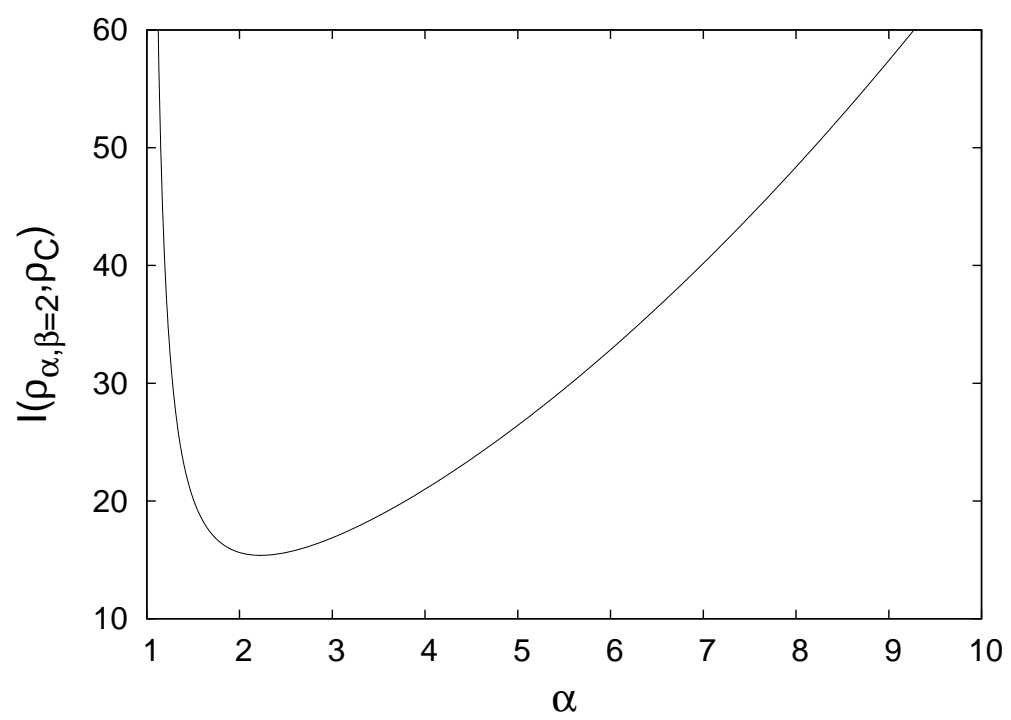

Fig. 2. $I\left(\rho_{\alpha, \beta=2}, \rho_{C}\right)$ for the Jacobi-like class as a function of $\alpha$.

at $\alpha=2$. The minimum is rapidly reached, and then the relative Fisher information increases in a monotonic, smooth and parabolic form.

\section{Fisher's information of special functions from their differential equations}

In this section we define the Fisher information of the probability density associated to the solutions of the second-order differential equation (1), and express it in terms of the expectation values of its coefficients $a(x)$ and $b(x)$. Then, we apply the subsequent general results to various special functions.

The spreading of the solutions $u(x) \in L_{2}(\Delta)$ of the differential equation (1) all over their domain of definition $\Delta=(a, b)$ can be measured by means of the Fisher information $I[u]$ of the associated probability density $\rho(x)=u^{2}(x)$, which is defined by

$$
I[u]=\int_{\Delta} \frac{\left[\rho^{\prime}(x)\right]^{2}}{\rho(x)} d x=4 \int_{\Delta}\left[u^{\prime}(x)\right]^{2} d x
$$

Two integrations by parts and Eq. (1) give that

$$
I[u]=4\langle b(x)\rangle-2\left\langle a^{\prime}(x)\right\rangle+\left[2 a(x) u^{2}(x)+4 u(x) u^{\prime}(x)\right]_{\Delta},
$$

where the expectation values are, following (5),

$$
\langle f(x)\rangle=\int_{\Delta} f(x) u^{2}(x) d x,
$$


and the boundary term (b.t.)

$$
[f(x)]_{\Delta} \equiv[f(x)]_{a}^{b}=f(b)-f(a) .
$$

The Fisher information of (the solutions of the) generalized equation of hypergeometric-type (2) can then be obtained by use of the functions $a(x)=$ $\tilde{\tau}(x) / \sigma(x)$ and $b(x)=\tilde{\sigma}(x) / \sigma^{2}(x)$ in Eq. (10), obtaining that

$$
I[u]=\left\langle\frac{4 \tilde{\sigma}-2 \tilde{\tau}^{\prime} \sigma+2 \tilde{\tau} \sigma^{\prime}}{\sigma^{2}}\right\rangle+\text { b.t. }
$$

Let us now apply these expressions for the calculation of the Fisher information of some special functions satisfying some differential equations which are frequently encountered in various scientific fields.

(a) Non-relativistic wavefunctions. The motion of a particle in the quantummechanical potential $V(x),-\infty<x<+\infty$, is governed by the Schrödinger equation

$$
-\frac{1}{2} u^{\prime \prime}(x)+V(x) u(x)=E u(x)
$$

where $E$ denotes the total energy of the particle in some appropriate units. This equation can be rewritten in the form (1) with $a(x)=0$ and $b(x)=$ $2[E-V(x)]$. Then it is straightforward to find from Eq. (10) the Fisher information for the square integrable of physically admissible solutions of this equation (which are the stationary wavefunctions of the quantummechanical states of the particle), namely

$$
I[u]=8[E-\langle V(x)\rangle] .
$$

This expression shows that the Fisher information is, the factor 8 apart, the kinetic energy of the particle as it was previously known by different means $[33,41,43,50]$.

(b) Airy functions. The motion of a particle subject to a liner potential is governed (see e.g. [47]) by the Schrödinger equation of Airy type

$$
u^{\prime \prime}(x)-x u(x)=0,
$$

where $u(x)=\operatorname{Ai}(x), x \in\left[\beta_{n},+\infty\right)$, being $\beta_{n}=a_{n / 2+1}^{\prime}$ and $a_{(n+1) / 2}$ for even and odd $n$, respectively, the roots of the derivative $\operatorname{Ai}^{\prime}(x)$ and the fuction $\operatorname{Ai}(x)$. Then, the comparison of Eqs. (1) and (11) and the use of Eq. (10) allows one to obtain the value

$$
I[u]= \begin{cases}\frac{4}{3}\left(a_{\frac{n}{2}+1}^{\prime}\right)^{2}\left[\operatorname{Ai}\left(a_{\frac{n}{2}+1}^{\prime}\right)\right]^{2}, & \text { even } \mathrm{n} \\ -\frac{4}{3} a_{\frac{n+1}{2}}\left[\operatorname{Ai}^{\prime}\left(a_{\frac{n+1}{2}}\right)\right]^{2}, & \text { odd } \mathrm{n}\end{cases}
$$


for the Fisher information of Airy functions $\operatorname{Ai}(x)$. It is worthy to remark that this local quantity is analytically accessible, in contrast to the global information-theoretic quantities such that the Shannon information of this system which is only known in the asymptotic $(n \rightarrow \infty)$ region [47].

(c) Rakhmanov-type functions. They are defined $[32,36]$ by

$$
u_{\nu}(x)=y_{\nu}(x) \sqrt{\omega(x)}
$$

where $y(x)=y_{\nu}(x)$ are functions of hypergeometric type, so that they satisfy the equation (3)

$$
\sigma(x) y^{\prime \prime}+\tau(x) y^{\prime}+\lambda_{\nu} y(x)=0
$$

where $\sigma(x)$ and $\tau(x)$ are polynomials with degrees $\leq 2$ and $\leq 1$, respectively; $\nu$ is a root of the equation

$$
\lambda_{\nu}+\nu \tau^{\prime}+\frac{1}{2} \nu(\nu-1) \sigma^{\prime \prime}=0
$$

and $\omega(x)$ is the solution of the Pearson equation

$$
[\sigma(x) \omega(x)]^{\prime}=\tau(x) \omega(x)
$$

It is straightforward to show [32] that the functions $u_{\nu}(x)$ fulfill a secondorder differential equation of the form (1) with the coefficients

$$
\begin{aligned}
& a(x)=\frac{\sigma^{\prime}(x)}{\sigma(x)} \\
& b(x)=\frac{1}{4 \sigma^{2}(x)}\left\{2 \sigma(x)\left(2 \lambda_{\nu}-\tau^{\prime}+\sigma^{\prime \prime}\right)-\left(\tau(x)-\sigma^{\prime}(x)\right)^{2}\right\} .
\end{aligned}
$$

Moreover, remark that the corresponding differential equation is a generalized equation of hypergeometric type. The associated probability density is the normalized-to-unity function

$$
\rho_{\nu}(x)=\left|u_{\nu}(x)\right|^{2}=y_{\nu}^{2}(x) \omega(x),
$$

to be referred as Rakhmanov-type density in honor to the mathematician who has shown $[38,51]$ that for the case $\nu$ equal to an integer, this density controls the asymptotic $(n \rightarrow \infty)$ behaviour of the polynomial ratio $y_{n+1}(x) / y_{n}(x)$. The Fisher information of this density function can easily be obtained by the substitution of the values (16) and (17) into the expression (10), which gives

$$
I\left[u_{\nu}\right]=\left\langle\frac{\left[\sigma^{\prime}(x)\right]^{2}+2 \tau(x) \sigma^{\prime}(x)-\tau^{2}(x)}{\sigma^{2}(x)}\right\rangle+\left(4 \lambda_{\nu}-2 \tau^{\prime}(x)\right)\left\langle\frac{1}{\sigma(x)}\right\rangle+\text { b.t. }
$$


where the boundary term is

$$
\left[4 y_{\nu}(x) y_{\nu}^{\prime}(x) \omega(x)+\frac{2 \tau(x)}{\sigma(x)} y_{\nu}^{2}(x) \omega(x)\right]_{\Delta}
$$

The most popular instances of Rakhmanov-type functions are the Hermite, Laguerre and Jacobi functions given by

$$
\begin{aligned}
& u_{H}(x)=\hat{H}_{n}(x) e^{-\frac{1}{2} x^{2}} ; \Delta=(-\infty,+\infty) \\
& u_{L}(x)=\hat{L}_{n}^{(\alpha)}(x) \sqrt{x^{\alpha} e^{-x}} ; \Delta=[0, \infty) \\
& u_{J}(x)=\hat{P}_{n}^{(\alpha, \beta)}(x) \sqrt{(1-x)^{\alpha}(1+x)^{\beta}} ; \Delta=[-1,+1],
\end{aligned}
$$

respectively, where $\hat{H}_{n}(x), \hat{L}_{n}^{(\alpha)}(x)$ and $\hat{P}_{n}^{(\alpha, \beta)}(x)$ denote the Hermite, Laguerre and Jacobi orthonormal polynomials [36].

The use of Eq. (18) for these three orthonormal functions allows us to obtain the values

$$
\begin{gathered}
I\left[\hat{H}_{n}\right] \equiv I\left[u_{H}\right]=4 n+2, \\
I\left[\hat{L}_{n}^{(\alpha)}\right] \equiv I\left[u_{L}\right]= \begin{cases}\frac{(2 n+1) \alpha+1}{\alpha^{2}-1}, & \alpha>1 \\
4 n+1, & \alpha=0, \\
+\infty, & \alpha \in(-1,+1] / 0\end{cases}
\end{gathered}
$$

and

$$
\begin{aligned}
I\left[P_{n}^{(\alpha, \beta)}\right] \equiv I\left[u_{P}\right]= & \frac{(2 n+\alpha+\beta+1}{4(n+\alpha+\beta-1)} \\
& \times\left[n(n+\alpha+\beta-1)\left(\frac{n+\alpha}{\beta+1}+2+\frac{n+\beta}{\alpha+1}\right)\right. \\
& \left.+(n+1)(n+\alpha+\beta)\left(\frac{n+\alpha}{\beta-1}+2+\frac{n+\beta}{\alpha-1}\right)\right], \\
& \alpha, \beta>1,
\end{aligned}
$$

for the Fisher information of the Rakhmanov density of the classical orthogonal polynomials (Hermite, Laguerre and Jacobi) already found from their corresponding hypergeometric-type equation of the form (3) with a different technique [48]. The Hermite value (22) was also derived by Hall [28] in calculating the Fisher information of the position and momentum stationary harmonic oscillator quantum-mechanical states, because their corresponding wavefunctions are Hermite functions. 


\section{Relative Fisher information of special functions}

In this section we consider the relative Fisher information of the probability density associated to the solutions of the second-order differential equation (1) with respect to an arbitrary non-negative weight function $\omega(x)$, and we express it by means of the expectation values of the coefficients $a(x)$ and $b(x)$. Then, we use these general results for various special functions relevant in applied mathematics and $D$-dimensional quantum physics.

For the solutions $u(x) \in L_{2}(\Delta)$ of Eq. (1) one can define the probability density $\rho(x)=u^{2}(x)$ as before. Then, following (8), we analyze the relative separation of this density with respect to an arbitrary non-negative weight function $\omega(x)$ by means of the Fisher information between them, namely,

$$
I_{\omega}[u]=\int_{\Delta} \frac{\left[\rho^{\prime}(x)\right]^{2}}{\rho(x)} \omega(x) d x=4 \int_{\Delta}\left[u^{\prime}(x)\right]^{2} \omega(x) d x .
$$

Remark that this expression boils down to Eq. (7) for uniformity on $\Delta$, so that $I_{1}[u]=I[u]$.

Let us now calculate the generalized Fisher information $I_{\omega}[u]$ from the differential equation (1). This can be done in a similar way as described in Section 3 for $I[u]$. We have obtained the following value

$$
I_{\omega}[u]=4\langle b(x)\rangle-2\left\langle a^{\prime}(x)\right\rangle+2\left\langle\frac{\omega^{\prime \prime}(x)-\omega^{\prime}(x) a(x)}{\omega(x)}\right\rangle+\text { b.t. },
$$

with the boundary term

$$
\text { b.t. }=\left\{4 u(x) u^{\prime}(x) \omega(x)+2 u^{2}(x)\left[\omega(x) a(x)-\omega^{\prime}(x)\right\}_{\Delta}\right.
$$

and the expectation value

$$
\langle f(x)\rangle_{\omega}=\int_{\Delta} f(x)|u(x)|^{2} \omega(x) d x .
$$

Then, the relative Fisher information of the (solutions of the) generalized equation of hypergeometric-type (2) with respect to a weight function $\omega(x)$ can be obtained from Eqs. (26)-(27) with the coefficients $a(x)=\tilde{\tau}(x) / \sigma(x)$ and $b(x)=\tilde{\sigma}(x) / \sigma^{2}(x)$; the result is

$$
I_{\omega}[u]=\left\langle\frac{4 \tilde{\sigma}-2 \tilde{\tau}^{\prime} \sigma+2 \tilde{\tau} \sigma^{\prime}}{\sigma^{2}}\right\rangle+2\left\langle\frac{\omega^{\prime \prime}-\omega^{\prime} \tilde{\tau} / \sigma}{\omega}\right\rangle+\text { b.t. }
$$

These expressions are used in the following to the calculation of the relative Fisher information of various special functions which naturally arise in 
numerous physicomathematical problems with standard and non-standard dimensions.

(a) $D$-dimensional nonrelativistic wavefunctions. The equation of motion of a particle moving in a $D$-dimensional spherically-symmetric potential $V_{D}(r)$ is the Schrödinger equation

$$
\left[-\frac{1}{2} \vec{\nabla}_{D}^{2}+V_{D}(r)\right] \psi(\vec{r})=E \psi(\vec{r})
$$

where $\vec{\nabla}_{D}^{2}$ denotes the Laplacian operator, and $\{E, \psi(\vec{r})\}$ are the eigenvalues and the corresponding eigenfunctions which charaterize the wavefunctions of the particle with the position vector $\vec{r} \equiv\left(r, \Omega_{D-1}\right)$ in polar hyperspherical coordinates: the hyperradius $r$ and the hyperangular coordinates $\Omega_{D-1} \equiv$ $\left(\theta_{1}, \theta_{2}, \ldots, \theta_{D-1}\right)$. This problem is known (see e.g. $\left.[1,7]\right)$ to be separable, so that

$$
\psi_{E, l,\{\mu\}}(\vec{r})=R_{E, l}(r) \mathcal{Y}_{l,\{\mu\}}\left(\Omega_{D-1}\right),
$$

where $R(r)$ and $\mathcal{Y}\left(\Omega_{D-1}\right)$ denote the hyperradial wavefunction and the wellknown hyperspherical harmonics [1] which are characterized by the hyperangular quantum numbers $\left(l \equiv \mu_{1}, \mu_{2}, \ldots, \mu_{D-1}\right) \equiv(l,\{\mu\})$. Moreover, $R(r)$ satisfies the following $D$-dimensional radial Schrödinger equation $[1,5]$

$$
-\frac{1}{2} R^{\prime \prime}-\frac{D-1}{2 r} R^{\prime}+\left[\frac{l(l+D-2)}{r^{2}}+V_{D}(r)\right] R=E R .
$$

Then, the associated quantum-mechanical probability density of the particle is

$$
\rho(\vec{r})=\left|\psi_{E, l,\{\mu\}}(\vec{r})\right|^{2}=R_{E, l}^{2}(r)\left|\mathcal{Y}_{l,\{\mu\}}\left(\Omega_{D-1}\right)\right|^{2},
$$

whose Fisher information is given by

$$
I[\rho]=\int \rho(\vec{r})\left[\vec{\nabla}_{D} \ln \rho(\vec{r})\right]^{2} d^{D} r=\int \frac{\left|\vec{\nabla}_{D} \rho(\vec{r})\right|^{2}}{\rho(\vec{r})} d^{D} r
$$

where the solid angle element is

$$
d^{D} r=r^{D-1} d r d \Omega_{D-1} ; d \Omega_{D-1}=\left(\prod_{j=1}^{D-2}\left(\sin \theta_{j}\right)^{2 \alpha_{j}} d \theta_{j}\right) d \theta_{D-1},
$$

with $2 \alpha_{j}=D-j-1$. The expression (29) can be separated out as

$$
I[\rho]=I_{\omega}[R]+I[\mathcal{Y}]
$$

where

$$
I[\mathcal{Y}]:=\int_{S_{D}} \frac{\left.\left.\left|\vec{\nabla}_{D-1}\right| \mathcal{Y}_{l,\{\mu\}}\left(\Omega_{D-1}\right)\right|^{2}\right|^{2}}{\left|\mathcal{Y}_{l,\{\mu\}}\left(\Omega_{D-1}\right)\right|^{2}} d \Omega_{D-1}
$$


denotes the angular part of the Fisher information, which has been recently calculated [10], and

$$
I_{\omega}[R]:=\int_{0}^{\infty} \frac{\left[\frac{d}{d r} R^{2}(r)\right]^{2}}{R^{2}(r)} \omega(r) d r ; \omega(r)=r^{D-1}
$$

which describes the radial part of $I[\rho]$, to be referred as relative radial Fisher information of the particle, which is our main purpose here. The comparison between (1) and (28) together with the formula (26) allows us to obtain that

$$
I_{\omega}[R]=8\left[E-\left\langle V_{D}\right\rangle\right]-4 l(l+D-2)\left\langle r^{-2}\right\rangle,
$$

which shows the explicit connection of the relative Fisher information, the kinetic energy and the so-called fictitious centrifugal potential [49].

(b) Hypergeometric-type functions $y_{\nu}(x)$. As already pointed out, these functions satisfy Eqs. (3) or (13) and (14). The relative Fisher information of these functions with respect to the Pearson weight function $\omega(x)$ given by Eq. (15) is defined by the relative Fisher information between the associated probability density $\rho(x)=\left|y_{\nu}(x)\right|^{2}$ and $\omega(x)$. This quantity, according to Eq. (25), is

$$
I_{\omega}\left[y_{\nu}\right]=4 \int_{\Delta}\left[y_{\nu}^{\prime}(x)\right]^{2} \omega(x) d x .
$$

Since Eq. (3) or (13) has the form (1) with $a(x)=\tau(x) / \sigma(x)$ and $b(x)=$ $\lambda_{\nu} / \sigma(x)$, the relative Fisher information can be expressed as

$$
I_{\omega}\left[y_{\nu}\right]=2\left\langle\frac{\left(2 \sigma^{\prime}-\tau\right) \sigma^{\prime}}{\sigma^{2}}\right\rangle+2\left(2 \lambda_{\nu}-\sigma^{\prime \prime}\right)\left\langle\frac{1}{\sigma}\right\rangle+\text { b.t. }
$$

where the boundary term is

$$
\left[4 y_{\nu} y_{\nu}^{\prime} \omega+2 \frac{\sigma^{\prime}}{\sigma}\left|y_{\nu}\right|^{2} \omega\right]_{\Delta}
$$

To illustrate the usefulness of these general expression, let us apply it to the classical orthogonal polynomials (Hermite, Laguerre and Jacobi). We have found the values

and

$$
\begin{gathered}
I_{\omega}\left(\hat{H}_{n}\right)=8 n, \\
I_{\omega}\left(\hat{L}_{n}^{(\alpha)}\right)=\frac{4 n}{\alpha+1} ; \alpha>-1,
\end{gathered}
$$

$$
I_{\omega}\left(\hat{P}_{n}^{(\alpha, \beta)}\right)=\frac{n(n+1+\alpha+\beta)(2 n+1+\alpha+\beta)(\alpha+\beta+2)}{(\alpha+1)(\beta+1)} ; \alpha, \beta>-1,
$$

for the Fisher information of the densities associated to the orthonormal Hermite $\hat{H}_{n}(x)$, Laguerre $\hat{L}_{n}^{(\alpha)}(x)$ and Jacobi $\hat{P}_{n}^{(\alpha, \beta)}(x)$ with respect to the weight functions $\omega_{H}(x)=\exp \left(-x^{2}\right), \omega_{L}(x)=x^{\alpha} e^{-x}$ and $\omega_{J}(x)=(1-$ $x)^{\alpha}(1+x)^{\beta}$, respectively. 


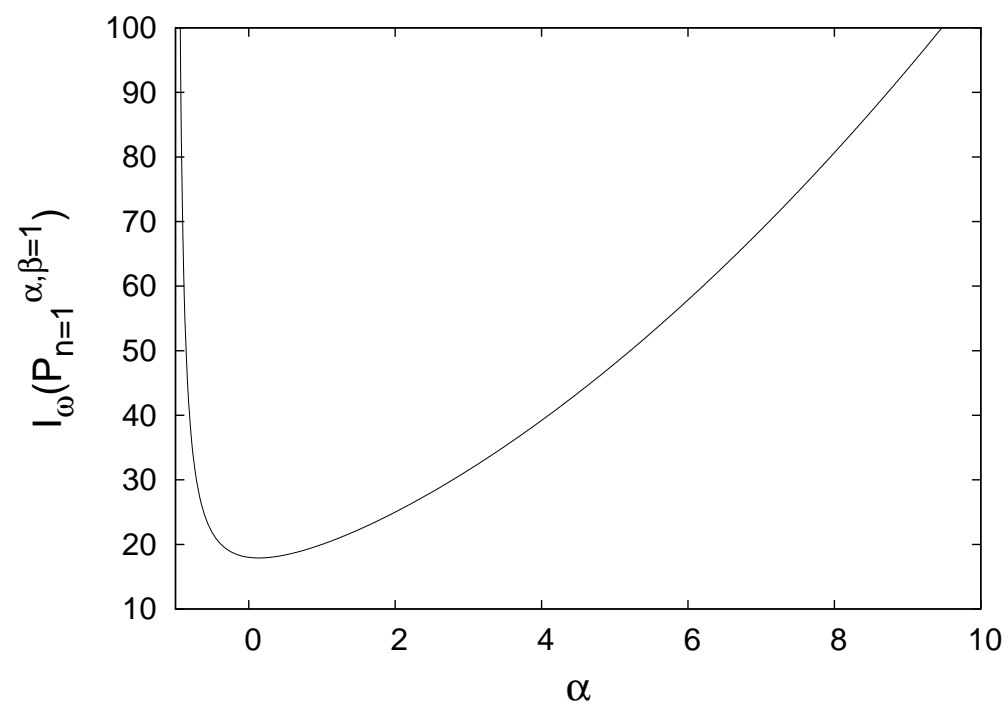

Fig. 3. $I_{\omega}\left(\hat{P}_{n=1}^{(\alpha, \beta=1)}\right)$ as a function of $\alpha$.

In Figure 3 the relative Fisher information $I_{\omega}\left(\hat{P}_{n}^{(\alpha, \beta)}\right)$ is represented as a function of $\alpha$ for $n=1, \beta=1$. Notice that this quantity increases as $\alpha$ tends to -1 and to infinity. In these cases, both the density and the weight function are pushed to +1 or -1 , respectively, increasing their gradient content. However, since the density for $n=1$ has one zero in the interval of orthogonality, the gradient content increases faster for this function than for the weight function, yielding a larger relative Fisher information between them.

\section{Conclusions and open problems}

The analytical determination of spreading quantifiers of special functions of applied mathematics and mathematical physics beyond the familiar variance is the goal of a mathematical programme which includes the calculation of the explicit and asymptotic expressions of the global (e.g. Shannon, Renyi, Tsallis) and local (Fisher) information-theoretic measures of the probability distributions associated with them. The performance of this programme has far reaching consequences for numerous scientific fields. However, except for the asymptotic determination of the Shannon entropy of Airy [47] and Bessel $[12,13]$ functions, most attempts have been done for orthogonal polynomials where the asymptotics of Shannon and Renyi entropies is reasonably well controlled (see [11] for the state-of-the-art up until 2001). It is based on the known algebraic properties of these functions other than the differential equation which they satisfy.

Contrary to the Shannon entropies, where explicit expressions are unreachable up until now, save for Chebyshev and other subfamilies of Gegenbauer 
polynomials, the Fisher information [48] (and the Cramer-Rao information plane [16]) have recently been determined for the classical orthogonal polynomials (Hermite, Laguerre, Jacobi) in terms of their degrees and characterizing parameters by means of a differential-equation-based methodology.

In this paper we have extended these efforts in a two-fold way. First, we present a simpler differential-equation-based methodology to calculate the Fisher information of special functions which satisfy Eqs. (1), (2) or (3); so, not necessarilly orthogonal polynomials but including them. We have illustrated the general resulting expression (given in terms of the expectation values of the differential-equation coefficients) for one-dimensional quantum-mechanical non-relativistic wavefunctions, Airy functions and Rakhmanov-type functions, obtaining the known Fisher information of the classical orthogonal polynomials [48] as a particular and checking case.

Second, we have discussed the not-so-well-known notion of relative Fisher information of two probability distributions to calculate the Fisher information of a special function fulfilling Eq. (1), with respect to a non-negative function. The usefulness of the subsequent results is illustrated (i) in some problems of non-relativistic $D$-dimensional quantum physics, where the relative Fisher information is shown to be related to some fundamental physical quantities of the involved system, and (ii) to calculate the relative Fisher information of general hypergeometric-type functions, which has allowed us to find explicit expressions for this notion when applied to classical orthogonal polynomials.

\section{Acknowledgements}

We are very grateful for partial support to Junta de Andalucía (under the grants FQM-0207 and FQM-481), Ministerio de Educación y Ciencia (under the project FIS2005-00973), and the European Research Network NeCCA (under the project INTAS-03-51-6637). PSM acknowledges the support of Ministerio de Educación y Ciencia under the program FPU.

\section{References}

[1] J. Avery, Hyperspherical Harmonics and Generalized Sturmians, Kluwer, Dordrecht, 2000.

[2] V. G. Bagrov, D. M. Gitman, Exact Solutions of Relativistic Wavefunctions, Kluwer Acad. Publ., Dordrecht, 1990. 
[3] J. L. Cardoso, R. Álvarez-Nodarse, Recurrence relations for radial wavefunctions for the nth-dimensional oscillators and hydrogenlike atoms, J. Phys. A: Math. Gen. 36 (2003) 2055-2068.

[4] J. L. Cardoso, C. M. Fernández, R. Álvarez-Nodarse, On properties of hypergeometric type-functions, Adv. Diff. EquationsAccepted.

[5] A. Chatterjee, Large- $\mathrm{N}$ expansions in quantum mechanics, atomic physics and some O(N) invariant systems, Phys. Rep. 186 (1990) 249.

[6] T. M. Cover, J. A. Thomas, Elements of Information Theory, Wiley, N.Y., 1991.

[7] J. S. Dehesa, R. González-Férez, P. Sánchez-Moreno, Fisher informationbased uncertainty relation, Cramer-Rao inequality and kinetic energy for $d$ dimensional central potentials, J. Phys. A: Math. Theor. 40 (2007) 1845-1856.

[8] J. S. Dehesa, S. López-Rosa, B. Olmos, R. J. Yáñez, Information measures of hydrogenic systems, Laguerre polynomials and spherical harmonics, J. Comput. Appl. Math. 179 (2005) 185-194.

[9] J. S. Dehesa, S. López-Rosa, B. Olmos, R. J. Yáñez, Fisher information of ddimensional hydrogenic systems in position and momentum spaces, J. Math. Phys. 47 (2006) 052104.

[10] J. S. Dehesa, S. López-Rosa, R. J. Yáñez, Information-theoretic measures of hyperspherical harmonics, J. Math. Phys. 48 (2007) 043503.

[11] J. S. Dehesa, A. Martínez-Finkelshtein, J. Sánchez-Ruiz, Quantum information entropies and orthogonal polynomials, J. Comput. Appl. Math. 133 (2001) 2346.

[12] J. S. Dehesa, A. Martínez-Finkelshtein, V. N. Sorokin, Short-wave asymptotics of the information entropy of a circular membrane, Int. J. Bifurcation \& Chaos 12 (2002) 2387-2392.

[13] J. S. Dehesa, A. Martínez-Finkelshtein, V. N. Sorokin, Asymptotics of information entropies of some Toda-like potentials, J. Mathem. Phys. 44 (2003) $36-47$.

[14] J. S. Dehesa, A. Martínez-Finkelshtein, V. N. Sorokin, Information-theoretic measures for Morse and Pöschl-Teller potentials, Mol. Phys. 104 (2006) 613622.

[15] J. S. Dehesa, B. Olmos, R. J. Yáñez, Parameter-based Fisher's information of orthogonal polynomials, J. Comput. Appl. Math.Accepted.

[16] J. S. Dehesa, P. Sánchez-Moreno, R. J. Yáñez, Cramer-Rao information plane of orthogonal hypergeometric polynomials, J. Comput. Appl. Math. 186 (2006) $523-541$.

[17] J. S. Dehesa, R. J. Yáñez, Fundamental recurrence relations of functions of hypergeometric type and their derivatives of any order, Il Nuovo Cimento 109B (1994) 711-723. 
[18] J. S. Dehesa, R. J. Yáñez, M. Pérez-Victoria, A. Sarsa, Nonlinear characterization for functions of hypergeometric type and their derivatives of an order, J. Math. Anal. Appl. 184 (1994) 35-43.

[19] J. S. Dehesa, R. J. Yáñez, A. Zarzo, J. A. Aguilar, New linear relationships of hypergeometric-type functions with applications to orthogonal polynomials, Rendiconti di Matematica, Serie VII, Roma 13 (1993) 661-671.

[20] W. N. Everitt, K. H. Kwon, L. L. Littlejohn, R. Wellman, Orthogonal polynomial solutions of linear ordinary differential equations, J. Comput. Appl. Math. 133 (2001) 85-109.

[21] R. A. Fisher, Theory of statistical estimation, Proc. Cambridge Phil. Soc. 22 (1925) 700-725, reprinted in Collected Papers of R.A. Fisher, edited by J.H. Bennet (University of Adelaide Press, South Australia), 1972, 15-40.

[22] S. Flügge, Practical Quantum Mechanics, Springer, Berlin, 1971.

[23] B. R. Frieden, Fisher information, disorder, and the equilibrium distributions of physics, Phys. Rev. A 41 (1990) 4265.

[24] B. R. Frieden, Science from Fisher Information, Cambridge University Press, Cambridge, 2004.

[25] B. R. Frieden, A. Plastino, A. R. Plastino, B. H. Soffer, Fisher-based thermodynamics: its Legendre transform and concavity properties, Phys. Rev. E 60 (1999) 48-53.

[26] R. González-Férez, J. S. Dehesa, Characterization of atomic avoided crossings by means of Fisher's information, Eur. Phys. J. D 32 (2005) 39-43.

[27] A. González-López, N. Kamran, P. Olver, Quasi-exact solvability, invited talk, in: AMS session n. 837, Springfield, USA, 20-21 March, 1992.

[28] M. J. W. Hall, Quantum properties of classical Fisher information, Phys. Rev. A 62 (2000) 012107.

[29] M. J. W. Hall, Exact uncertainty relations, Phys. Rev. A 64 (2001) 052103.

[30] I. P. Hamilton, R. A. Mosna, L. D. Site, Classical kinetic energy, quantum fluctuation terms and kinetic energy functionalsPreprint.

[31] L. L. Littlejohn, Orthogonal polynomial solutions to ordinary and partial differential equations, in: M. Alfaro, J. S. Dehesa, F. J. Marcellán, J. L. R. de Francia, J. Vinuesa (eds.), Orthogonal Polynomials and their Applications, Lecture Notes in Math. Vol. 1329, Springer-Verlag, Berlin, 1988.

[32] M. Lorente, Raising and lowering operators, factorization and differentialdifference operators of hypergeometric type, J. Phys. A: Math. Gen. 34 (2001) $569-588$.

[33] S. L. Luo, Fisher information, kinetic energy and uncertainty relation inequalities, J. Phys. A: Math. Gen. 35 (2002) 5181. 
[34] P. M. Morse, H. Feshbach, Methods of Theoretical Physics, McGraw-Hill, New York, 1953.

[35] A. Nagy, Fisher information in density functional theory, J. Chem. Phys 119 (2003) 9401-9405.

[36] A. F. Nikiforov, V. B. Uvarov, Special Functions in Mathematical Physics, Birkäuser-Verlag, Basel, 1988.

[37] A. Plastino, A. R. Plastino, B. H. Soffer, Fisher info and thermodynamics first law, Physica A 369 (2006) 432-438.

[38] E. A. Rakhmanov, On the asymptotics of the ratio of orthogonal polynomials, Math. USSR Sb. 32 (1977) 199-213.

[39] M. Reginatto, Derivation of the equations of nonrelativistic quantum mechanics using the principle of minimum Fisher information, Phys. Rev. A 58 (1998) $1775-1778$.

[40] E. Romera, J. C. Angulo, J. S. Dehesa, Fisher entropy and uncertaintylike relationships in many-body systems, Phys. Rev. A 59 (1999) 4064.

[41] E. Romera, J. S. Dehesa, Weizsäcker energy of many-electron systems, Phys. Rev. A 50 (1994) 256.

[42] E. Romera, J. S. Dehesa, The Fisher-Shannon information plane, an electron correlation tool, J. Chem. Phys. 120 (2004) 8906-8912.

[43] E. Romera, J. S. Dehesa, R. J. Yáñez, The Weizsäcker functional: some rigorous results, Int. J. Quantum Chem. 56 (1995) 627-632.

[44] E. Romera, P. Sánchez-Moreno, J. S. Dehesa, The Fisher information of singleparticle systems with a central potential, Chem. Phys. Lett. 414 (2005) 468.

[45] E. Romera, P. Sánchez-Moreno, J. S. Dehesa, Uncertainty relation for fisher information of d-dimensional single-particle systems with central potentials, J. Math. Phys. 47 (2006) 103504.

[46] N. Saad, R. L. Hall, H. Ciftci, Criterion for polynomial solutions to a class of linear differential equation of second order, J. Phys. A: Math. Gen. 39 (2006) 13445, see references herein.

[47] P. Sánchez-Moreno, R. J. Yáñez, V. S. Buyarov, Asymptotics of the information entropy of the Airy function, J. Phys. A: Math. Gen. 38 (2005) 9969-9978.

[48] J. Sánchez-Ruiz, J. S. Dehesa, Fisher information of orthogonal hypergeometric polynomials, J. Comput. Appl. Math. 182 (2005) 150-164.

[49] W. P. Schleich, J. P. Dahl, Dimensional enhancement of kinetic energies, Phys. Rev. A 65 (2002) 052109.

[50] S. B. Sears, R. G. Parr, U. Dinur, On the quantum-mechanical kinetic energy as a measure of the information in a distribution, Israel J. Chem. 19 (1980) $165-173$. 
[51] B. Simon, Ratio asymptotics and weak asymptotic measures for orthogonal polynomials on the real line, J. Approx. Th. 126 (2004) 198-217.

[52] A. Stam, Some inequalities satisfied by the quantities of information of Fisher and Shannon, Inform. Control 2 (1959) 105-112.

[53] A. Turbiner, Lie algebras and polynomials in one variable, J. Phys. A: Math. Gen. 25 (1992) L1087-L1093.

[54] W. van Assche, R. J. Yáñez, J. S. Dehesa, Entropy of orthogonal polynomials with Freud weights and information entropies of the harmonic oscillator potential, J. Math. Phys. 36 (1995) 4106-4118.

[55] C. Vignat, J. F. Bercher, Analysis of signals in the Fisher-Shannon information plane, Phys. Lett. A 312 (2003) 27-33.

[56] E. T. Whittaker, G. N. Watson, A Course of Modern Analysis, 4th. ed., Cambridge Univ. Press, Cambridge, 1927.

[57] A. D. Wyner, J. Ziv, On communication of analog data from a bounded source space, Bell Syst. Tech. J. (1969) 3139-3172.

[58] R. J. Yáñez, J. S. Dehesa, A. F. Nikiforov, The three-term recurrence relation and the differentiation formulas for hypergeometric-type functions, J. Math. Anal. Appl. 188 (1994) 855-866.

[59] R. J. Yáñez, J. S. Dehesa, A. Zarzo, Four-term recurrence relations of hypergeometric-type polynomials, Il Nuovo Cimento 109B (1994) 725-733.

[60] A. Zarzo, R. J. Yáñez, J. S. Dehesa, General recurrence and ladder relations of hypergeometric-type functions, J. Comput. Appl. Math.Accepted. 